\title{
MOTION PLANNING OF A NOVEL 2-DOF PARALLEL MANIPULATOR APPLIED AS DRIVING SIMULATOR OF THE WHEEL LOADER
}

\author{
J. KNAPCZYK* \\ State Higher Vocational School in Nowy Sącz \\ 33-300 Nowy Sącz, Staszica 1, POLAND \\ E-mail:j_kn@mech.pk.edu.pl \\ G. TORA \\ Cracow University of Technology \\ 31-864 Cracow, Al. Jana Pawła II 37, POLAND \\ E-mail: tora@mech.pk.edu.pl
}

\begin{abstract}
A novel parallel manipulator with 3 legs ( 2 actuated by linear actuators and one supporting pillar),which is applied in a wheel loader driving simulator, is proposed in this paper. The roll angle and the pitch angle of the platform are derived in closed-form of functions of the variable lengths of two actuators. The linear velocity and acceleration of the selected point and angular velocity of the moving platform are determined and compared with measurement results obtained in the respective point and in the body of the wheel loader. The differences between the desired and actual actuator displacements are used as feedback to compute how much force to send to the actuators as some function of the servo error. A numerical example with a proposed mechanism as a driving simulator is presented.
\end{abstract}

Key words: simulator, wheel loader driving, motion planning.

\section{Introduction}

A parallel manipulator with 6 DOF, called Gough-Stewart platform, is used as a basic mechanism for advanced motion simulators. A driving simulator can be used to replicate situations, which are dangerous for human, and to study the influence of the driver fatigue on his reactions (Garrett and Best, 2013; Lozia, 2008).

A vehicle driving simulator used for training of vehicle drivers and wheel machine operators can be based on the RSSR-SS spatial mechanism. A driving simulator requires real-time calculation of vehicle dynamics in response to driver's input, such as steering maneuver, and accelerator or brake pedal operation. On the contrary, dynamic analysis involves a large amount of calculation, and it is difficult to realize in realtime with a high degrees simulator model. One of the reasons of difficulty is that the equation of motion of this simulator are represented as differential equations, so the calculation of the manipulator Jacobian is required in each step of control (Zhang and Zhang, 2013).

\section{Platform mechanism used as motion simulator of wheel loader}

A mechanism shown in Fig.1 is selected for modelling (Tora, 2012) and simulation analysis, as a compromise solution from the point of view of its functionality and simplicity. The mechanism is driven by two linear actuators. The suspension mechanism for the operator seat that can generate vertical oscillations

\footnotetext{
* To whom correspondence should be addressed
} 
is separate from the simulator. The simulator mechanism contains a movable platform $p$ suspended by three legs with three spherical joints with centers respectively at points $B_{1}, B_{2}$ and $B_{3}$. Two legs of the platform mechanism are designed with two rockers 2 and 3 joined with the machine frame $r$ by two revolute joints $\left(A_{2}\right.$ and $\left.A_{3}\right)$. The third leg is driven by a linear actuator 1 of the length $s_{1}$. The actuator cylinders $(1 c$ and $4 c)$ are joined with the frame $r$ by using two universal joints with center points respectively at $A_{1}$ and $A_{4}$. The actuator $4(4 t)$ is joined to the rocker 2 by using a spherical joint with a point center at $C_{4}$. The length of this actuator is $s_{4}$. The number of the mechanism mobility is 4 , where 2 degrees describe the global mobility and 2 degrees - the local mobility as angular displacements of the pistons ( $1 t$ and $4 t)$ around the longitudinal axes of cylinders $(1 c$ and $4 c$ ) have no influence on the main platform motion.

The mechanism that is composed of two rockers (2 and 3), one actuator (4) and platform $p$ with coupler $B_{2} B_{3}$, can be considered a planar mechanism, if the geometric conditions are satisfied, e.g., the joint centers $A_{4}, C_{4}, A_{2}, B_{2}, B_{3}$ and $A_{3}$ are situated in one plane, and axes of revolute joints $A_{2}$ and $A_{3}$ are parallel and perpendicular to this plane. The cost of technical manufacturing under these conditions can be smaller when the allowed tolerances are bigger, because some inaccuracies of this mechanism assembly have only a small influence on the symmetry of platform motion and the load transmission in the joint $C_{4}$. The mechanism is considered as parallel manipulator composed of the platform of spatial motion and 3 legs with joints: R-S, R$\mathrm{S}$, K-S, where: $\mathrm{R}$ - revolute joint, $\mathrm{S}$ - spherical joint, $\mathrm{K}$ - universal joint.

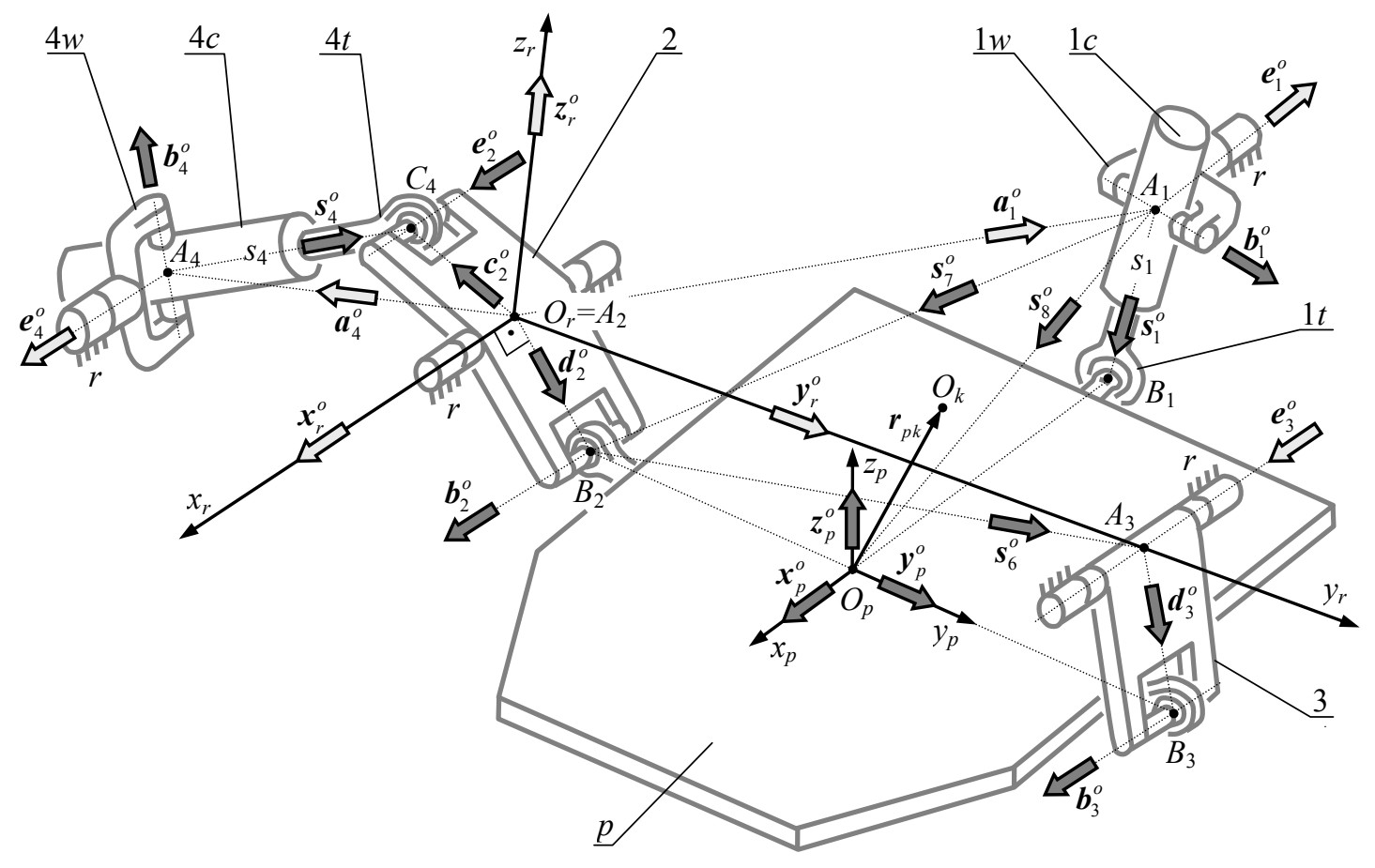

Fig.1. The platform mechanism as generator of the cab motion. Nomenclature: $r$ - cab frame of the loader machine; $p$ - platform; 2, 3 - rockers; $1 w, 4 w$-yokes of universal joints; $1 c, 4 c$-actuator cylinders; $1 t, 4 \mathrm{t}-$ pistons of actuators.

When the mechanism is in the middle position (the lowest position of the platform) the actuators 1 and 4 do not carry the gravity force of the cab mass, and in other positions this force is mainly carried by the joints $A_{2}$ and $A_{3}$. Inaccuracies of the mechanism do not introduce any passive constrains, which can cause over load of kinematic pairs. The function of the mechanism simulator is to generate cab oscillations: around the longitudinal axis $\boldsymbol{x}_{p}$ (roll motion) and around the lateral axis $\boldsymbol{y}_{p}$ (pitch motion) and linear oscillations along the lateral axis. 


\section{Kinematics and dynamics of simulator mechanism}

The control of the simulator drive systems used the solution of the direct problem for the mechanism position. It is necessary to determine the platform orientation as a function of the actuator lengths $s_{1}, s_{4}$. The cab orientation is described by axis unit vectors of the platform reference system $\left\{\boldsymbol{x}_{p} \boldsymbol{y}_{p} \boldsymbol{z}_{p}\right\}$. Algorithms of successive determination of the unit vectors are presented in Fig.2.

An unknown unit vector is determined by using two following relationships. Using the first relationship the unknown unit vector is determined on the basis of two known unit vectors, additionally the closed loop condition for the vector triangle formed with all three unit vectors has to be satisfied. The second relationship determines the unknown unit vector, when two unit vectors and the dot products of each of these vectors with the unknown unit vector are known. A group of known unit vectors is described in the basis frame.

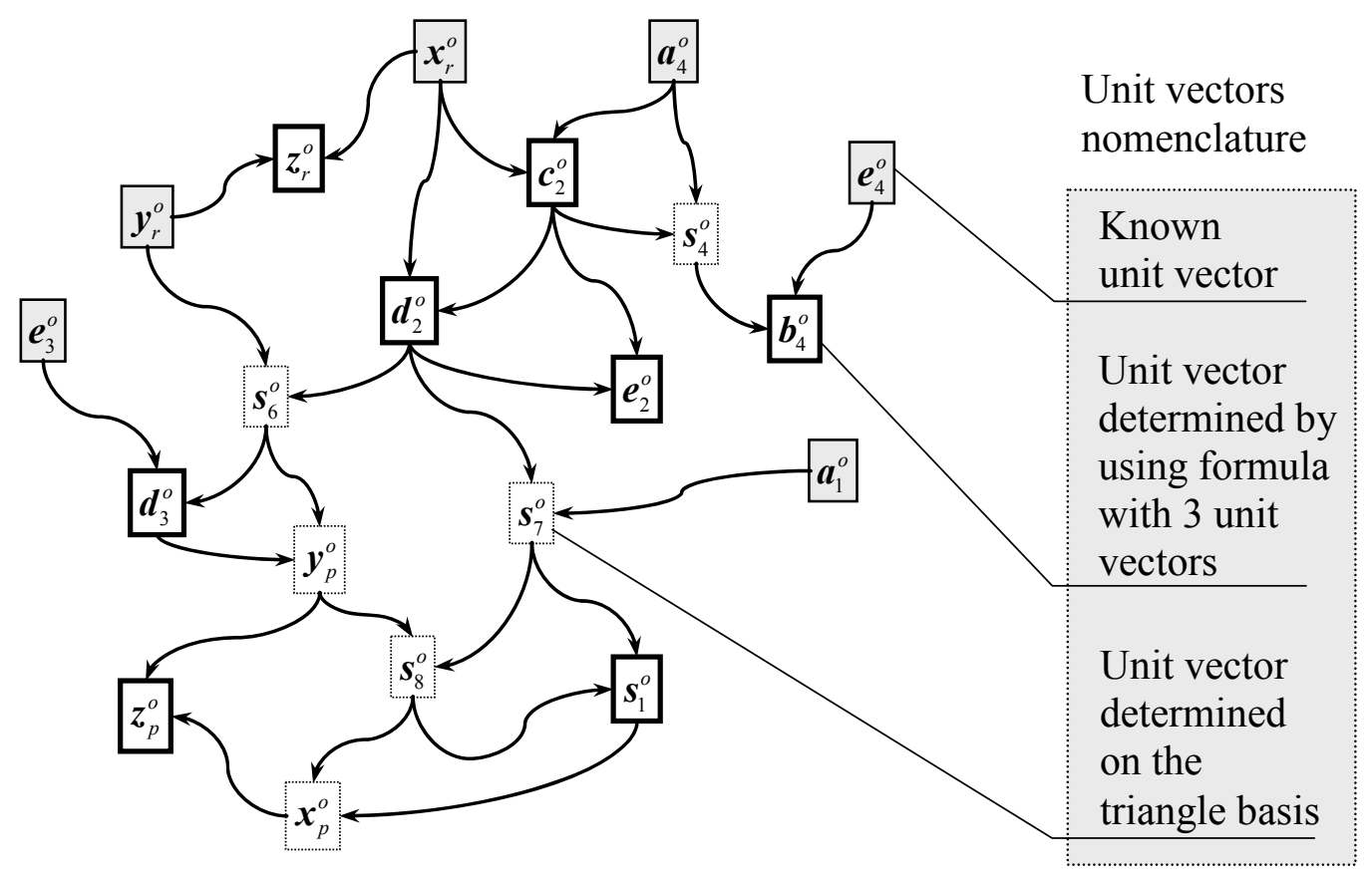

Fig.2. Algorithm for successive determination of the unit vectors.

On the basis of the determined unit vectors of the platform reference system it is possible to determine the orientation matrix of the platform with respect to the base reference system

$$
\boldsymbol{R}=\left[\begin{array}{ccc}
\boldsymbol{x}_{p}^{o} \cdot \boldsymbol{x}_{r}^{o} & \boldsymbol{y}_{p}^{o} \cdot \boldsymbol{x}_{r}^{o} & \boldsymbol{z}_{p}^{o} \cdot \boldsymbol{x}_{r}^{o} \\
\boldsymbol{x}_{p}^{o} \cdot \boldsymbol{y}_{r}^{o} & \boldsymbol{y}_{p}^{o} \cdot \boldsymbol{y}_{r}^{o} & \boldsymbol{z}_{p}^{o} \cdot \boldsymbol{y}_{r}^{o} \\
\boldsymbol{x}_{p}^{o} \cdot \boldsymbol{z}_{r}^{o} & \boldsymbol{y}_{p}^{o} \cdot \boldsymbol{z}_{r}^{o} & \boldsymbol{z}_{p}^{o} \cdot \boldsymbol{z}_{r}^{o}
\end{array}\right]
$$

The simulator can realize the programmed time functions of the platform velocities, when the inverse velocity problems are solved, and it is possible to determine the actuator velocities $\dot{s}_{1 o}, \dot{s}_{40}$. The angular velocity vector of the platform 


$$
\omega_{p}=\boldsymbol{J}_{\omega} \dot{\boldsymbol{s}}
$$

where: $\quad \dot{\boldsymbol{s}}=\left[\begin{array}{c}\dot{s}_{1} \\ \dot{s}_{4}\end{array}\right], \quad \boldsymbol{J}_{\omega}=\left[\begin{array}{ll}\boldsymbol{h}_{1} & \boldsymbol{h}_{4}\end{array}\right], \quad \boldsymbol{h}_{1}=\frac{\boldsymbol{y}_{p}^{o}}{r_{1 y}}, \quad \boldsymbol{h}_{4}=\frac{\boldsymbol{x}_{p}^{o}}{r_{4 x}}+\frac{\boldsymbol{y}_{p}^{o}}{r_{4 y}}+\frac{\boldsymbol{z}_{p}^{o}}{r_{4 z}}, \quad r_{1 y}=b_{1}\left(\boldsymbol{z}_{p}^{o} \cdot \boldsymbol{s}_{1}^{o}\right)$,

$$
\begin{aligned}
& r_{4 x}=\frac{b_{23}}{\left[\frac{d_{3}}{r_{3}}\left(\boldsymbol{e}_{3}^{o} \times \boldsymbol{d}_{3}^{o}\right)-\frac{d_{2}}{r_{2}}\left(\boldsymbol{x}_{r}^{o} \times \boldsymbol{d}_{2}^{o}\right)\right] \cdot \boldsymbol{z}_{p}^{o}}, r_{4 y}=\frac{r_{1 y}}{\left[\frac{b_{1}}{r_{4 z}} \boldsymbol{y}_{p}^{o}-\frac{d_{2}}{r_{2}}\left(\boldsymbol{x}_{r}^{o} \times \boldsymbol{d}_{2}^{o}\right)-\frac{b_{2}}{r_{4 x}} \boldsymbol{z}_{p}^{o}+\frac{b_{2}}{r_{4 z}} \boldsymbol{x}_{p}^{o}\right] \cdot \boldsymbol{s}_{1}^{o}}, \\
& r_{4 z}=\frac{b_{23}}{\left[\frac{d_{2}}{r_{2}}\left(\boldsymbol{x}_{r}^{o} \times \boldsymbol{d}_{2}^{o}\right)-\frac{d_{3}}{r_{3}}\left(\boldsymbol{e}_{3}^{o} \times \boldsymbol{d}_{3}^{o}\right)\right] \cdot \boldsymbol{x}_{p}^{o}}, \quad r_{3}=\frac{c_{2} d_{3}}{d_{2}} \frac{\left[\left(\boldsymbol{e}_{3}^{o} \times \boldsymbol{d}_{3}^{o}\right) \cdot \boldsymbol{y}_{p}^{o}\right]\left[\left(\boldsymbol{x}_{r}^{o} \times \boldsymbol{c}_{2}^{o}\right) \cdot \boldsymbol{s}_{4}^{o}\right]}{\left(\boldsymbol{x}_{r}^{o} \times \boldsymbol{d}_{2}^{o}\right) \cdot \boldsymbol{y}_{p}^{o}}, \\
& r_{2}=c_{2}\left[\left(\boldsymbol{x}_{r}^{o} \times \boldsymbol{c}_{2}^{o}\right) \cdot \boldsymbol{s}_{4}^{o}\right]
\end{aligned}
$$

$b_{1}=O_{p} B_{1}, b_{23}=B_{2} B_{3}, d_{2}=A_{2} B_{2}, d_{3}=A_{3} B_{3}, c_{2}=A_{2} C_{4}$ - dimensions of the mechanism.

When angular velocities of the platform are given, then the simulator controller has to calculate the instant linear velocities of the actuators by using the following formula

$$
\left[\begin{array}{c}
\dot{s}_{1} \\
\dot{s}_{4}
\end{array}\right]=\left[\begin{array}{ll}
\boldsymbol{h}_{1} \cdot \boldsymbol{x}_{r}^{o} & \boldsymbol{h}_{4} \cdot \boldsymbol{x}_{r}^{o} \\
\boldsymbol{h}_{1} \cdot \boldsymbol{y}_{r}^{o} & \boldsymbol{h}_{4} \cdot \boldsymbol{y}_{r}^{o}
\end{array}\right]^{-1}\left[\begin{array}{c}
\omega_{p x} \\
\omega_{p y}
\end{array}\right] .
$$

The vector of linear velocity for the platform point $O_{k}$

$$
\boldsymbol{v}_{O_{k}}=\left(\boldsymbol{\omega}_{2} \times \boldsymbol{d}_{2}^{o}\right) d_{2}+\boldsymbol{\omega}_{p} \times\left(b_{2} \boldsymbol{y}_{p}^{o}+\boldsymbol{r}_{p k}\right)
$$

where $\quad \omega_{2}=\frac{\dot{s}_{4}}{r_{2}} \boldsymbol{x}_{r}^{o}, \quad b_{2}=O_{p} B_{2}, \quad \boldsymbol{r}_{p k}=\overrightarrow{O_{p} O_{k}}$.

Substituting relation (3.2) into Eq.(3.4) we can obtain

$$
\boldsymbol{v}_{O_{k}}=\boldsymbol{J}_{v} \dot{\boldsymbol{s}}
$$

where

$$
\boldsymbol{J}_{v}=\left[\begin{array}{ll}
\boldsymbol{k}_{1} & \boldsymbol{k}_{4}
\end{array}\right], \quad \boldsymbol{k}_{1}=\boldsymbol{h}_{1} \times\left(b_{2} \boldsymbol{y}_{p}^{o}+\boldsymbol{r}_{p k}\right), \quad \boldsymbol{k}_{4}=\frac{d_{2}}{r_{2}}\left(\boldsymbol{x}_{r}^{o} \times \boldsymbol{d}_{2}^{o}\right)+\boldsymbol{h}_{4} \times\left(b_{2} \boldsymbol{y}_{p}^{o}+\boldsymbol{r}_{p k}\right) .
$$

By using Eq.(3.5) it is possible to determine the linear velocity of the platform point $O_{k}$ on the basis of the instant velocities of the actuators. 
The solution of the inverse dynamic problem for the simulator mechanism is formulated as determination of the actuator driving forces that can generate the cab planning motion. The principle of virtual work is used with Newton-Euler equation (Cervantes-Sanchez, 2011; Tsai, 2000)

$$
\dot{\boldsymbol{s}}^{T} \boldsymbol{F}_{s}+m_{p} \boldsymbol{v}_{O_{k}}^{T}\left(\boldsymbol{g}-\boldsymbol{a}_{O_{k}}\right)-\boldsymbol{\omega}_{p}^{T}\left(\boldsymbol{R} \boldsymbol{I}_{p} \boldsymbol{R} \boldsymbol{\varepsilon}_{p}+\tilde{\boldsymbol{\omega}}_{p} \boldsymbol{R} \boldsymbol{I}_{p} \boldsymbol{R} \boldsymbol{\omega}_{p}\right)=0
$$

where: $\boldsymbol{F}_{S}=\left[\begin{array}{ll}F_{1} & F_{4}\end{array}\right]^{T}$ - vector of drive forces; $\boldsymbol{v}_{O_{k}}, \boldsymbol{a}_{O_{k}}$ - vectors of velocity and acceleration of point $O_{k}$; $\boldsymbol{\omega}_{p}, \boldsymbol{\varepsilon}_{p}$ - vectors of angular velocity and acceleration of platform motion; $\tilde{\boldsymbol{\omega}}_{p}$ - matrix combined with vector $\boldsymbol{\omega}_{p} ; \boldsymbol{I}_{p},-$ matrix of platform inertia moments with respect to the platform reference system.

On the basis of Eqs (3.2), (3.5) and (3.6) the actuator drive forces can be determined in relation to the platform inertia and gravity forces

$$
\boldsymbol{F}_{s}=m_{p} \boldsymbol{J}_{v}^{T}\left(\boldsymbol{a}_{O_{k}}-\boldsymbol{g}\right)+\boldsymbol{J}_{\omega}^{T}\left(\boldsymbol{R} \boldsymbol{I}_{p} \boldsymbol{R} \boldsymbol{\varepsilon}_{p}+\tilde{\boldsymbol{\omega}}_{p} \boldsymbol{R} \boldsymbol{I}_{p} \boldsymbol{R} \boldsymbol{\omega}_{p}\right)
$$

\section{Measurements of the wheel loader motion}

Results of measurements of the wheel loader (YTOZL50D-11) motion are recorded and filtered in order to obtain the real ranges of the body oscillations that can be caused by machine courses in terrain conditions. The wheel base and the position coordinates of the Crossbow measurement block (at point $P_{1}$ ) are shown in Fig.3.

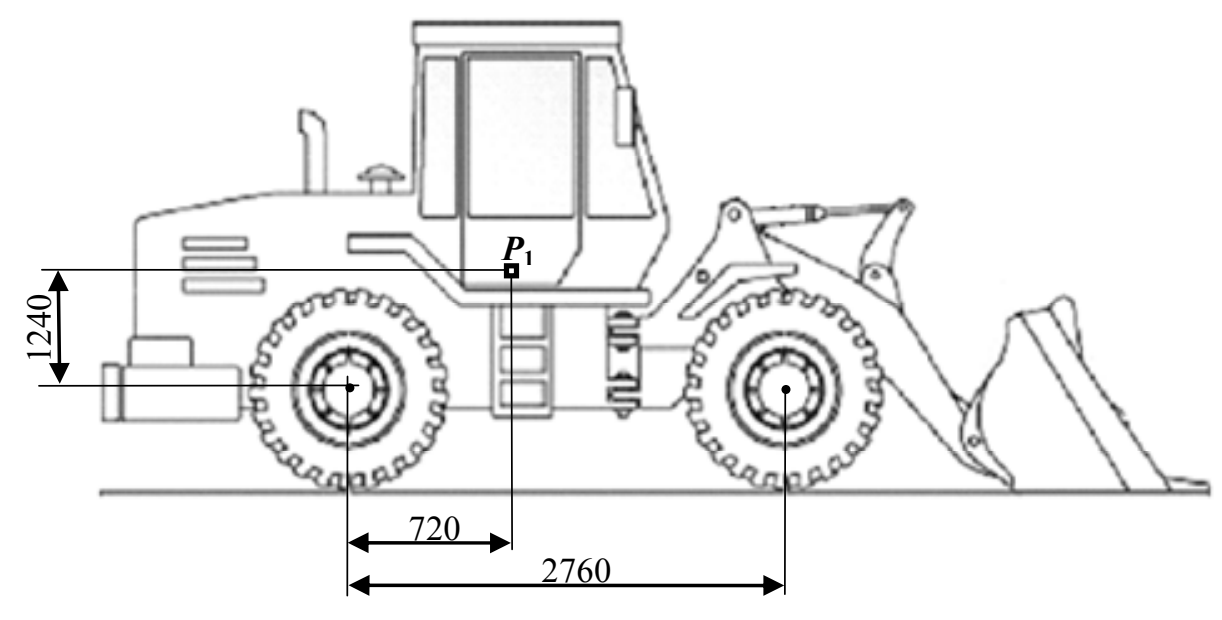

Fig.3. Position coordinates of the Crossbow block on the wheel loader (YTOZL50D-11).

Maximum values of the measured coordinates of the longitudinal and lateral accelerations: $a_{P_{1} x \max }=a_{P_{l} y \max } \approx 4,5 \mathrm{~m} / \mathrm{s}^{2}$. Maximum measured coordinates of the angular velocities in longitudinal and lateral direction are respectively: $\omega_{x \max }=4,5[\mathrm{rad} / \mathrm{s}] ; \omega_{y \max }=3,0[\mathrm{rad} / \mathrm{s}]$. The range of roll angle (around the longitudinal axis) $\Delta \alpha_{x}=12.6\left[^{o}\right]$ is something greater than the range of pitch angle (around the lateral axis) 
$\Delta \alpha_{y}=10.6\left[{ }^{\circ}\right]$, due to a some greater distance of the wheel axis $(2760[\mathrm{~mm}])$ than the distance of wheel planes $(2760[\mathrm{~mm}])$.

Frequency response of the cab angular velocity and linear acceleration shown (see diagrams in Figs 4 and 5) demonstrate that the important excitation range is to $8[\mathrm{~Hz}]$.
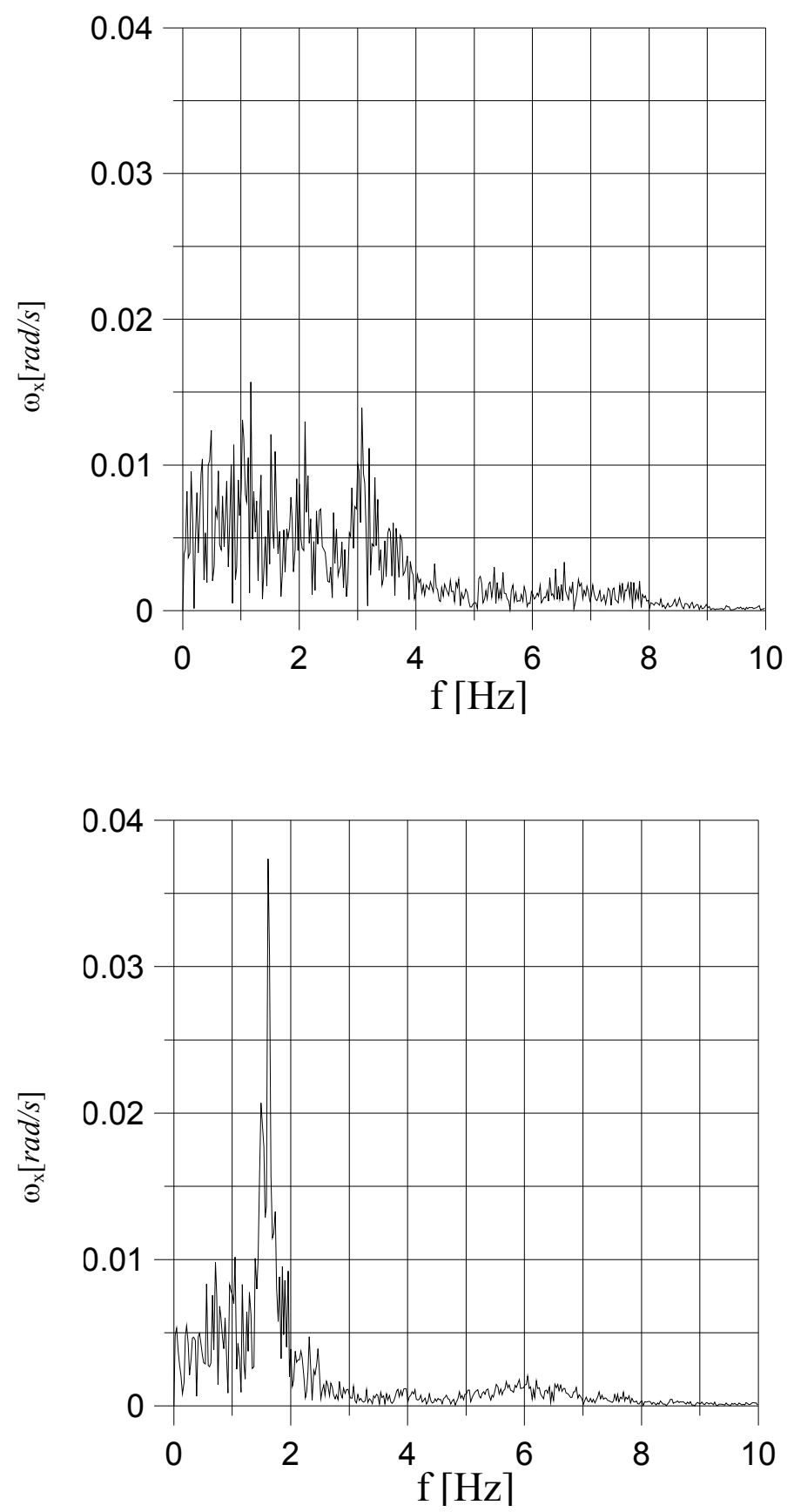

Fig.4. Components ( $x$ - roll, $y$-pitch) of the cab angular velocity vector with respect to excitation frequency. 

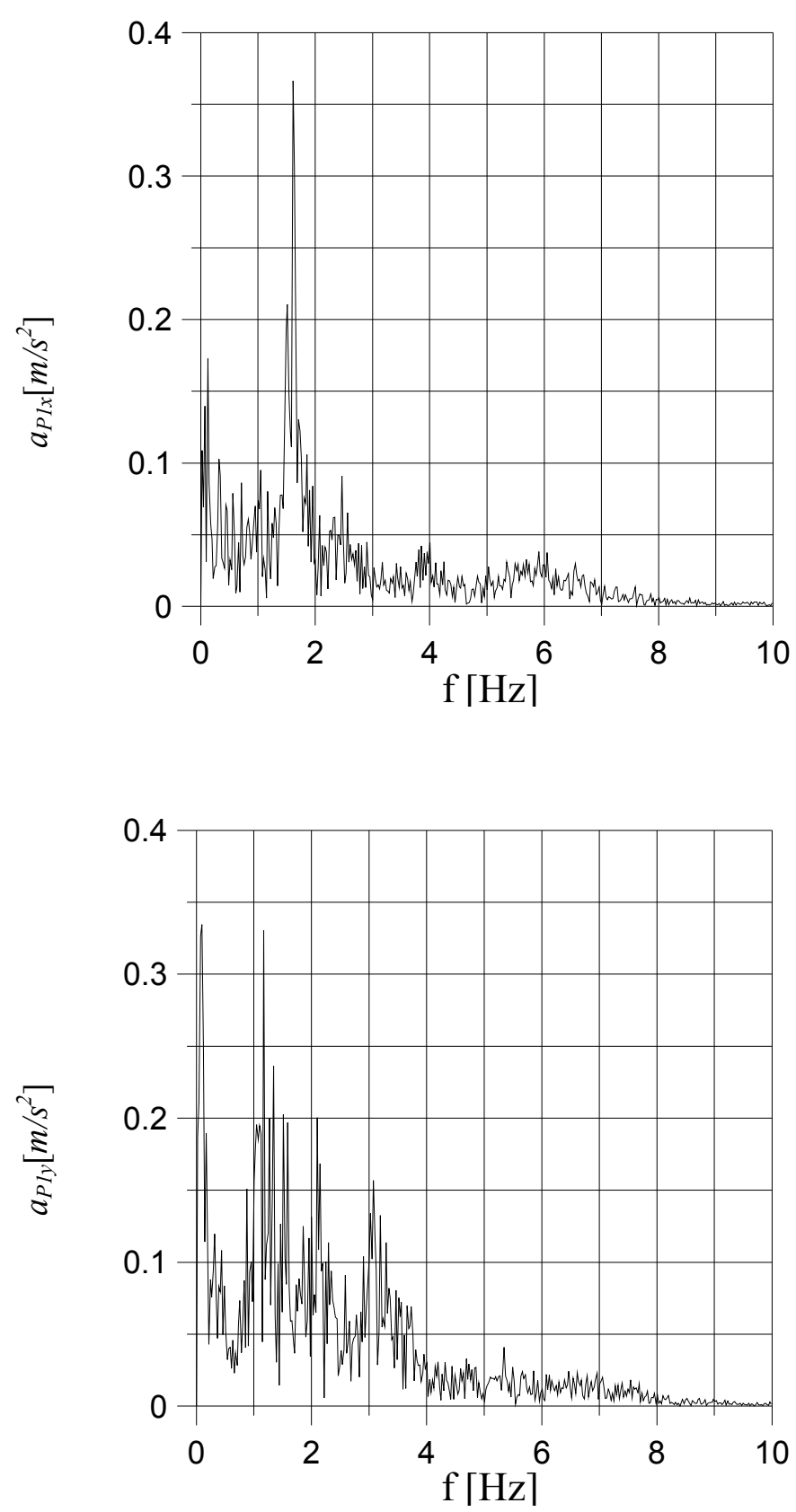

Fig.5. Longitudinal $(x)$ and lateral $(y)$ acceleration components of the point $P_{l}$ with respect to excitation frequency.

\section{Motion planning for simulator mechanism of the wheel loader}

Motion planning of the simulator mechanism necessitates a transformation from the measured and simulated motion of the wheel loader to the achievable platform motion. The measured coordinates of the cab angular velocity vector $\left(\omega_{x}\right.$ and $\left.\omega_{y}\right)$ are introduced as input signals. The actuators instantaneous 
velocities $\dot{s}_{1}$ and $\dot{s}_{4}$ are determined on the basis of relation (3.3). The vertical component $\left(\omega_{z}\right)$ of the instantaneous angular velocity of the platform, the velocity of the cab mass center $\left(v_{O_{k}}\right)$, and the actuator drive forces $F_{1}$ and $F_{4}$ are determined by using formulae (3.2), (3.4) and (3.6).

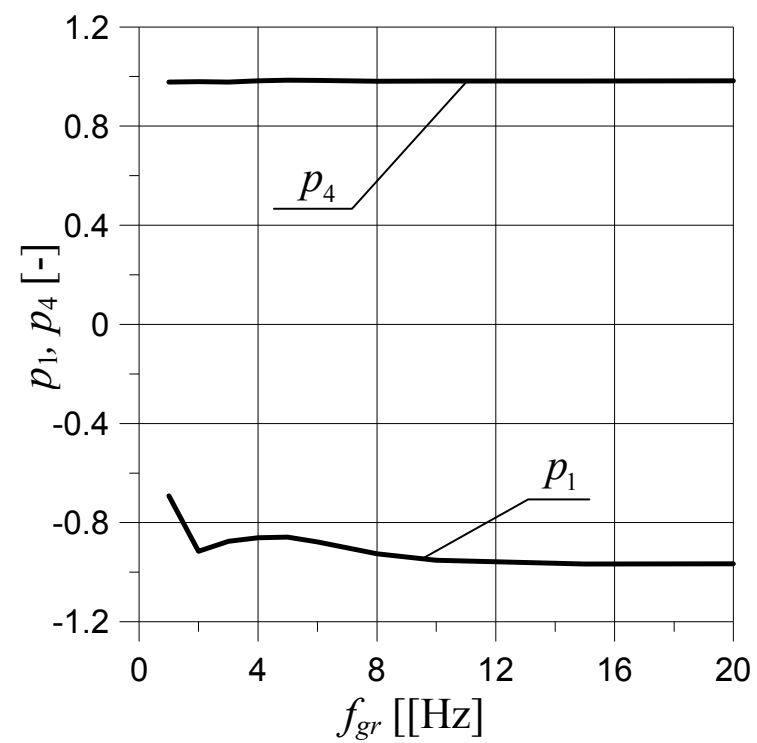

Fig.6. Pearson's correlation coefficients for the platform angular velocities and linear velocities in actuators 1 and 4.

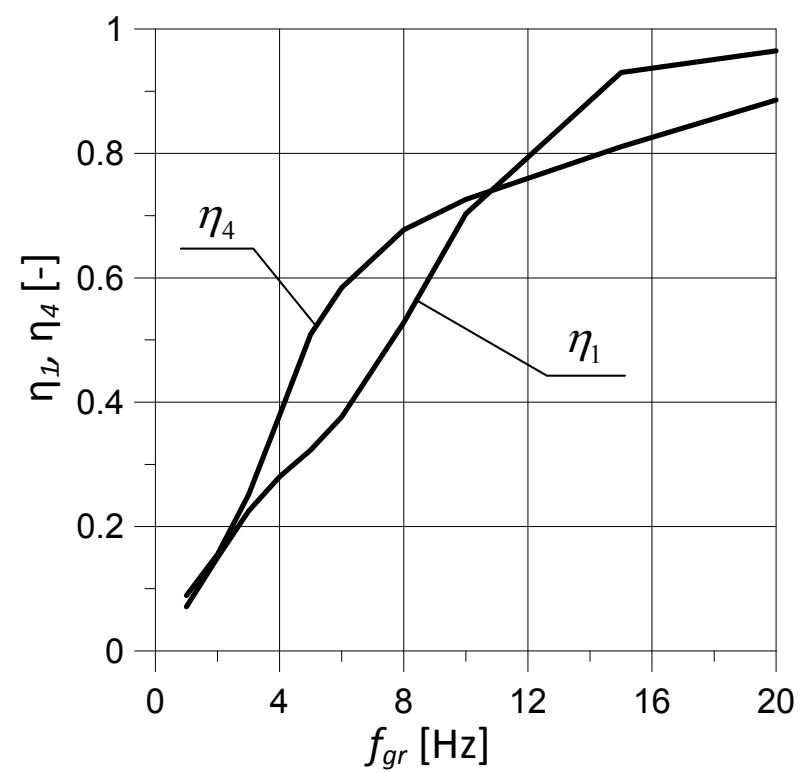

Fig.7. Power coefficients in relations to the limits of excitation frequency.

A low-pass filter with frequency limit $f_{g r}$ is situated before the signals $\omega_{x}$ and $\omega_{y}$. Dimensions of the mechanism are the same as in Tora (2012). 
Pearson's correlation coefficients are used to determine the degree of linearity in the relations between the platform angular velocity and the actuators linear velocities, where $p_{1}$ is used to describe the relation between the longitudinal component of the platform angular velocity ( $\omega_{x}$ - roll velocity) and the linear velocity of the rear actuator $\left(\dot{s}_{1}\right) ; p_{4}$ - the relation between the lateral component of the platform angular velocity ( $\omega_{y}$ - pitch velocity) and linear velocity of the lateral actuator $\left(\dot{s}_{4}\right)$. The coefficients $p_{1}$ and $p_{4}$ are presented as functions $f_{g r}$ in Fig.6. The influence of $f_{g r}$ on the used actuator power is illustrated in Fig.7, where the power coefficients are defined respectively as the ratio of the median power of actuators 1 and 4 to their median power without the filter; $\eta_{i}=N_{i, \text { med }, f_{g r}} / N_{i . m e d, f_{\infty}}(i=1,4)$.

\section{Conclusion}

A real-time analysis method for simulator dynamics is proposed to reduce the amount of calculation, and to obtain the sufficient accuracy for motion planning. The numerical effects are compared to experimental results obtained from the real wheel load machine.

The absolute values of Pearson's correlation coefficients are determined as close to unity. That confirms the majority of linear influences: the linear velocity of the rear actuator 1 on the roll angular velocity of the platform (in the lateral plane), and the linear velocity of the lateral actuator 4 on the pitch angular velocity (in the longitudinal plane). The power coefficients described in relation to the excitation frequency (see Fig.7) show that if the simulator excitation frequency is limited to $7[\mathrm{~Hz}]$, then the power consumption is smaller by about $50 \%$.

\section{Nomenclature}

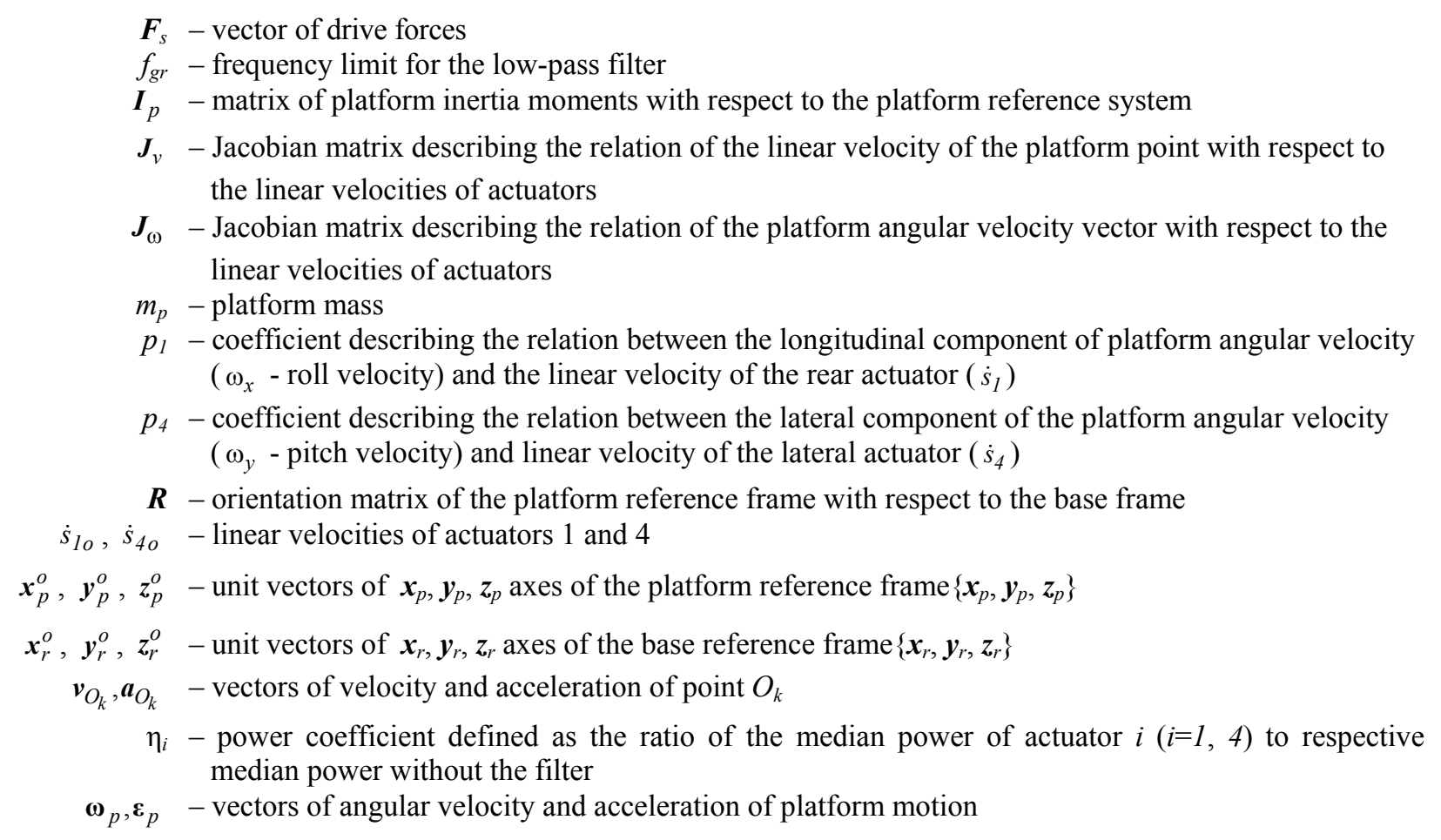




\section{References}

Cervantes-Sanchez J.J., Rico-Martínez J.M., Pacheco-Gutiérrez S. and Cerda-Villafañ G. (2011): Static analysis of spatial parallel manipulators by means of the principle of virtual work. - Robotics and Computer-Integrated Manufacturing, vol.26, Elsevier.

Garrett N.J.I. and Best M.C. (2013): Model predictive driving simulator motion cueing algorithm with actuator-based constraints. - Vehicle System Dynamics, Taylor and Francis.

Lozia Z. (2008): Car Driving Simulator. - Warsaw: WKŁ.

Tora G. (2012): Modelling of a platform mechanism of an active suspension system for the cab in a heavy machine. Monograph, Mechanics 416, Cracow 2012, Editorial Office Cracow University of Technology.

Tsai L.W. (2000): Solving the Inverse Dynamics of a Stewart-Gough Manipulator by the Principle of Virtual Work. Trans. ASME, Journal of Mechanical Design, vol.122.

Zhang C. and Zhang L. (2013): Kinematics analysis and workspace investigation of a novel 2-DOF parallel manipulator applied in vehicle driving simulator. - Robotics and Computer-Integrated Manufacturing 29, pp.113120, Elsevier.

Received: May 12, 2014

Revised: May 26, 2014 